\title{
Preclinical modeling and multimodality imaging of chronic myocardial infarction in minipigs induced by novel interventional embolization technique
}

\author{
Bo Tao ${ }^{1,2+}$, Haokao Gao ${ }^{2 \dagger}$, Minwen Zheng ${ }^{3}$, Zhonghua Luo ${ }^{3}$, Liwen Liư ${ }^{4}$, Wei Bai ${ }^{4}$, Jing Wang ${ }^{5}$, Daliang Liư ${ }^{5}$,
} Sai Ma², Zhenli Luo², Lei Gao', Yabin Wang ${ }^{1}$ and Feng Cao ${ }^{1 *}$

\begin{abstract}
Background: This study was designed to establish a chronic myocardial infarction (MI) model in minipigs with a novel coronary sequential balloons-sponge embolism technique.

Methods: Eighteen healthy minipigs $(25-30 \mathrm{~kg}$ ) were randomly divided into three groups for left anterior descending artery (LAD) occlusion: conventional balloon occlusion group (BO group, temporary balloon occlusion for 60 mins), half-balloon embolism group (HB group), and sequential balloon-balloon-sponge embolism group (BBS group, two half-balloons with one sponge as the embolism clot). The incidence of ventricular fibrillation (VF), total mortality, operating time, and vascular recanalization 3 months post-Ml was recorded and compared. Echocardiography, multimodality nuclear medical imaging, and histology staining were applied for the evaluation of infarction.

Results: Thirteen out of 18 minipigs survived after the operation, while 5 animals died with VF (3 in the BO group, 1 in the HB group, and 1 in the BBS group), with an $83.3 \%$ (5/6 minipigs) acute procedural survival rate in embolism groups. The operating duration was $60.0 \pm 0.5 \mathrm{mins}, 21.4 \pm 5.2 \mathrm{mins}$, and $31.2 \pm 4.7 \mathrm{mins}$ in the three groups, respectively. LAD recanalization was found in three animals of the HB group but none in the BBS group by angiography follow-up. The infarct sizes were more stable and larger in the HB group and BBS group than that in the $\mathrm{BO}$ group $(P<0.05, n=13)$.

Conclusions: The method of sequential balloons-sponge embolization could induce myocardial infarction with consistent and sustained embolization and gain higher operation success rate and better repeatability in minipigs, which holds a promising method for preclinical Ml study.
\end{abstract}

Keywords: Pig model, Myocardial infarction, Percutaneous coronary artery embolization, Balloon, Sponge

\section{Background}

Coronary artery disease and subsequent myocardial infarction (MI) are leading causes of morbidity and mortality in the world [1, 2]. Although advances in MI therapy have improved acute survival rates, such as percutaneous coronary intervention or coronary artery bypass graft, survivors are prone to development of chronic

\footnotetext{
* Correspondence: wind8828@gmail.com

${ }^{\dagger}$ Equal contributors

'Department of Cardiology, Chinese PLA General Hospital, Fuxing Street 28\#, Haidian District, Beijing 100853, China

Full list of author information is available at the end of the article
}

degenerative changes as a result of irreversible cardiomyocyte loss, extracellular matrix fibrosis, and pathological gene programming $[3,4]$. Large animal model of chronic MI with long-term postoperative survival is very important for studying the molecular and cellular changes following MI and testing novel therapies for prevention of chronic negative myocardial remodeling.

At present, MI modeling in most of preclinical experiments still apply surgical opening chest and coronary artery ligation or incision of the peripheral arteries for interventional approach and balloon occlusion of coronary flow, which are also supported by endotracheal intubation 
and assisted respiration. Left anterior descending coronary artery (LAD), especially mid-LAD was used for target artery [5-8]. The biggest advantage is that they maintain the consistency of infarct size and target vessel status. However, some issues and potential pitfalls should be recognized such as high incidence rate of malignant ventricular arrhythmias, less induced infarct size, longer operation time (generally required 5-6 h), and larger trauma. In this operation course, the blood flow interrupts completely and suddenly, resulting in a high incidence rate of malignant ventricular arrhythmias and mortality $[9,10]$. The temporary blockage of blood flow by balloon usually results in less induced infarct size and fast revascularization afterward, which is apt to overestimate the infarct size in the early stage [11]. In recent years, many methods of coronary artery embolization have been proposed and applied in large animals' MI modeling. But to our knowledge, these methods still need further optimization, due to limitations in high cost (spring coil) [12], difficulty in pinpointing embolism levels (micro-catheter injection of ethanol, micro-spheres embolization) [13-16], poor operational feasibility (sponge is hard to push) [17], and postoperative vascular recanalization (autologous blood clots, winding stent, spring coil) [18-20].

In the present study, we initiated a consistent and sustained coronary embolism model with high operation success rate and short operative time by the sequential balloons-sponge embolization in a large animal so as to lay a good foundation for further heart intervention. Furthermore, multimodality clinical imaging and histology staining were applied to evaluate the efficacy of the method compared with traditional modeling by interventional technology.

\section{Methods}

\section{Experimental protocols}

This study was performed at an Association for the Assessment and Accreditation of Laboratory Animal Care International-accredited large animal research facility. All animal procedures were conducted in conformity with the National Institutes of Health Guide for the Care and Use of Laboratory Animals. A total of 18 minipigs (female, weighing $25-30 \mathrm{~kg}$ ) were randomly allocated into three groups $(n=6$, each group) including balloon occlusion group (BO group), half-balloon embolism group (HB group), and sequential balloonballoon-sponge embolism group (BBS group, two halfballoons with one sponge as the embolism clot). Coronary artery occlusion or embolization was kept in the mid-LAD, immediately after the ostium of the second diagonal branch $[5,10,21,22]$. The following data were compared in three groups: the incidence of ventricular fibrillation (VF), total mortality, operation time of occlusion or embolization, and vascular recanalization at 3 months post-MI. MI in the BBS group was confirmed by electrocardiogram, echocardiography, and ${ }^{18} \mathrm{~F}$-FDG positron emission tomography/computed tomography (PET/CT) imaging. The combination evaluating methods of echocardiography, single photon emission computed tomography/computed tomography (SPECT/CT), and histology staining were applied to compare the infarct size in three groups.

\section{Common procedures of $\mathrm{MI}$ induction in three groups}

The three groups all went through the following six procedures including anesthesia, percutaneous femoral artery puncture, selective coronary angiography (CAG), conventional ischemic preconditioning and prevention of arrhythmia, balloon occlusion or embolization, and postoperative monitoring and treatment.

Briefly, animals were anesthetized with ketamine (20 $\mathrm{mg} / \mathrm{kg}, \mathrm{IM})$ and introduced intravenous catheters in marginal ear veins. Continuous infusion of propofol (0.003 $\mathrm{ml} / \mathrm{kg} / \mathrm{min})$ was administrated to maintain anesthesia. Animals were continuously monitored and inhaled oxygen $(3 \mathrm{~L} / \mathrm{min})$. The right or left femoral artery was identified, and a 6-Fr radial arterial introducer sheath (Terumo, Japan) was advanced over the guidewire in the femoral artery by percutaneous puncture. When it was necessary, ultrasound could be used to assist the puncture. Heparin sodium was given as a 150 $\mathrm{IU} / \mathrm{kg}$ bolus IV at the start of the procedure and continued at $50 \mathrm{IU} / \mathrm{kg} / \mathrm{h} \mathrm{IV}$ throughout the operation. Femoral artery angiography was performed (Fig. 1), and a 6-Fr guiding catheter (Judkins right 4.0 type) was advanced into the ostium of the coronary artery. Baseline selective coronary angiography and left ventricular angiography were performed, and the target blocking location was determined. Lidocaine $(0.03 \mathrm{mg} / \mathrm{kg} / \mathrm{min})$ was intravenously infused throughout the following procedures. In case of VF, nonsynchronized direct current defibrillation was performed at $360 \mathrm{~J}$. Along with the guidewire, the $1.5-2.5-\mathrm{mm}$ balloon catheter according to target vessel diameter was inflated and deflated three times in the mid-LAD as the ischemic preconditioning $[23,24]$. Each time, the balloon was inflated 6-8 atm for 5 mins with the interval of $1 \mathrm{~min}$. Subsequently, all animals were performed mid-LAD balloon occlusion or embolism procedure according to grouping. Finally, the arterial sheath was removed, followed by 30 mins oppression on the femoral artery puncture area. After the procedures, all the animals received antibiotics (penicillin $640 \mathrm{WU}$ once a day, for 3 days).

\section{Procedures of balloon occlusion or embolization Balloon occlusion group (Classic method of MI induction)}

After ischemic preconditioning, matched angioplasty balloon (with the diameter of targeted artery position) was advanced to the mid-LAD. The balloon was inflated 


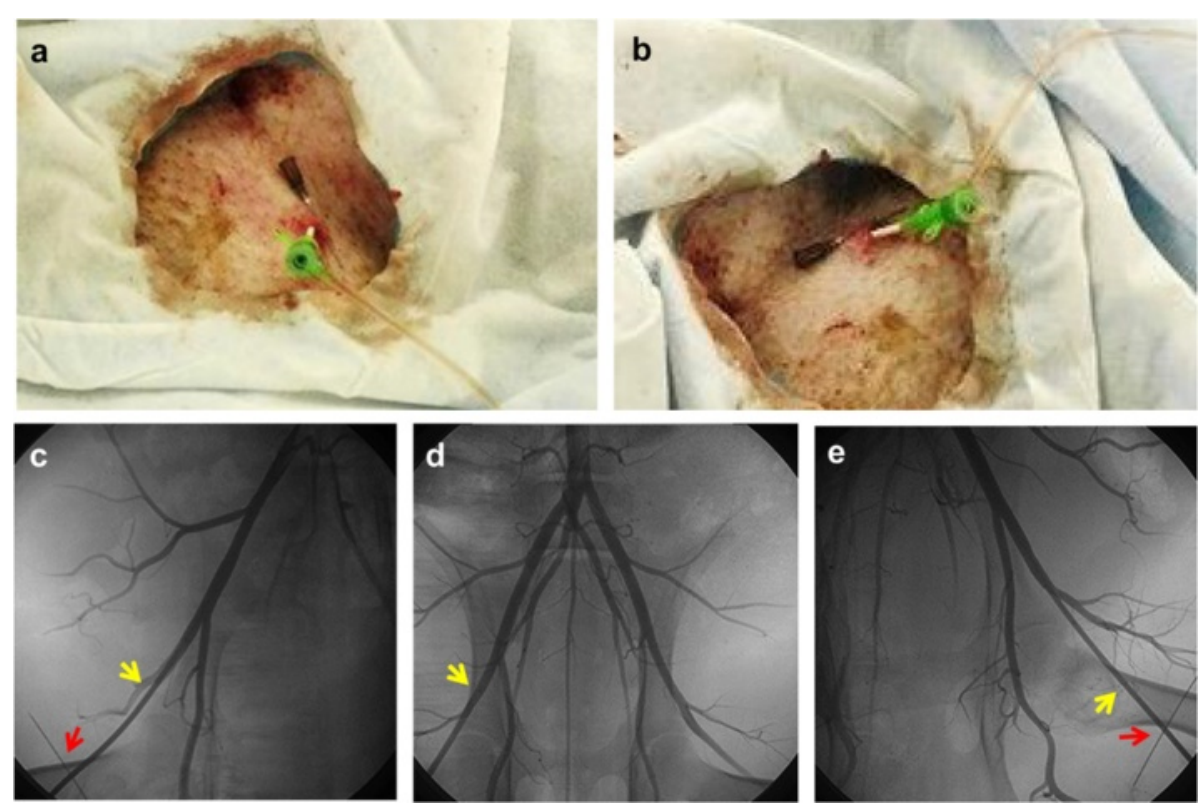

Fig. 1 Minipig femoral artery angiography in three groups. a, b Display arterial introducer sheath in the right and left femoral artery, respectively. c-e The angiography of right and left femoral artery and iliac artery, internal iliac artery, and abdominal aorta, respectively. The yellow arrows indicate the location of introducer sheath, and the red arrows indicate the location of skin puncture

at 6-8 atm for 60 mins to block the blood flow completely without affecting the second diagonal branch. After that, the balloon was deflated and withdrawn, and LAD was reperfused (Figs. $2 \mathrm{a}-\mathrm{d}$ and $3 \mathrm{a}-\mathrm{c}$ ).

\section{Half-balloon embolism group}

After ischemic preconditioning, two balloon catheters were used. The first inflated balloon was cut in the median, and the distal free half-balloon (about $10 \mathrm{~mm}$, half spindle type, withered shape, with the tip of a metal marker) was set along the guidewire. After that, the second balloon catheter was set along the guidewire and pushed $10 \mathrm{~mm}$ distal to the ostium of the second branch of LAD. Then, the guidewire was retraced to the second balloon catheter. During this process, there was no displacement in the distal half-balloon (with the metal

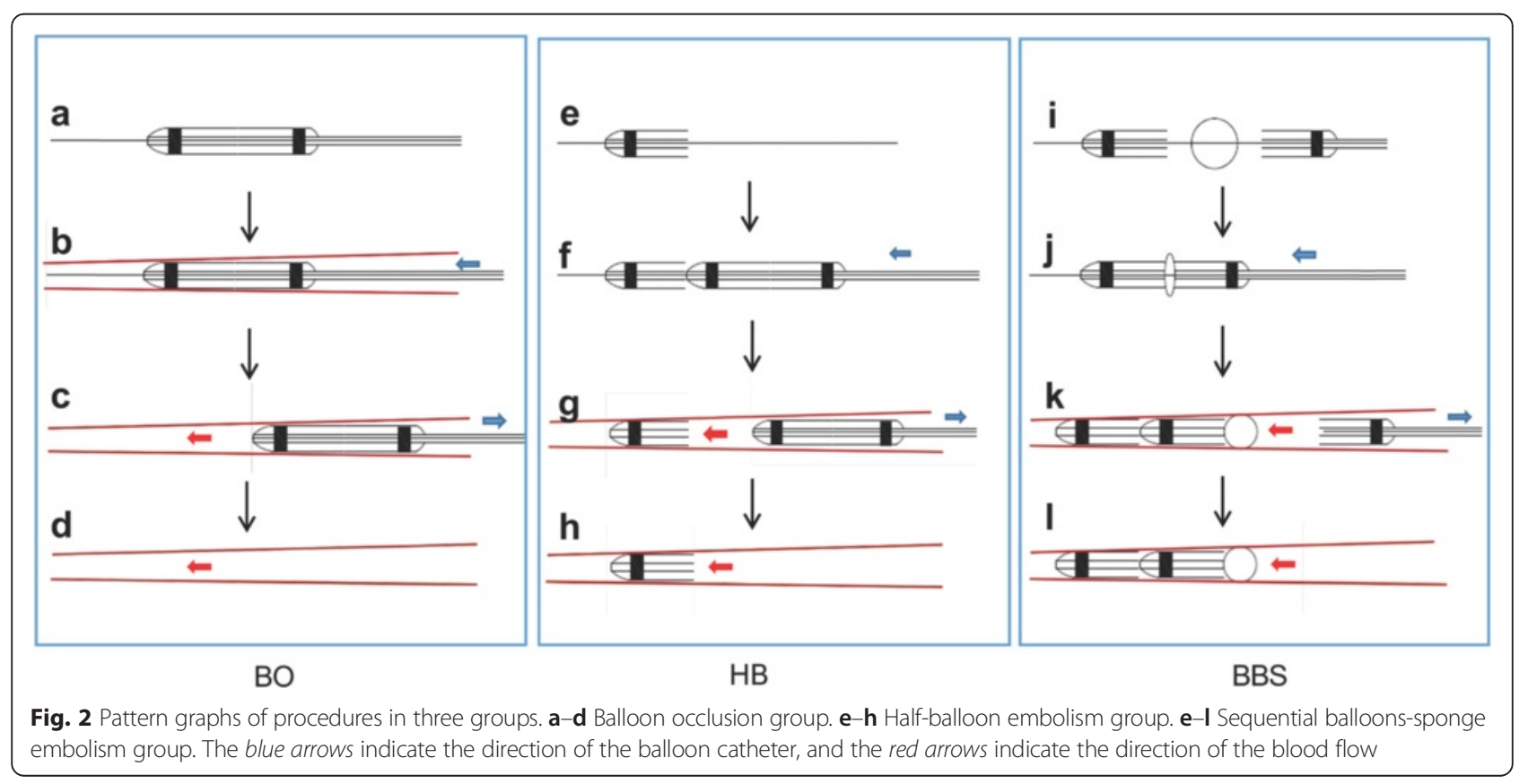




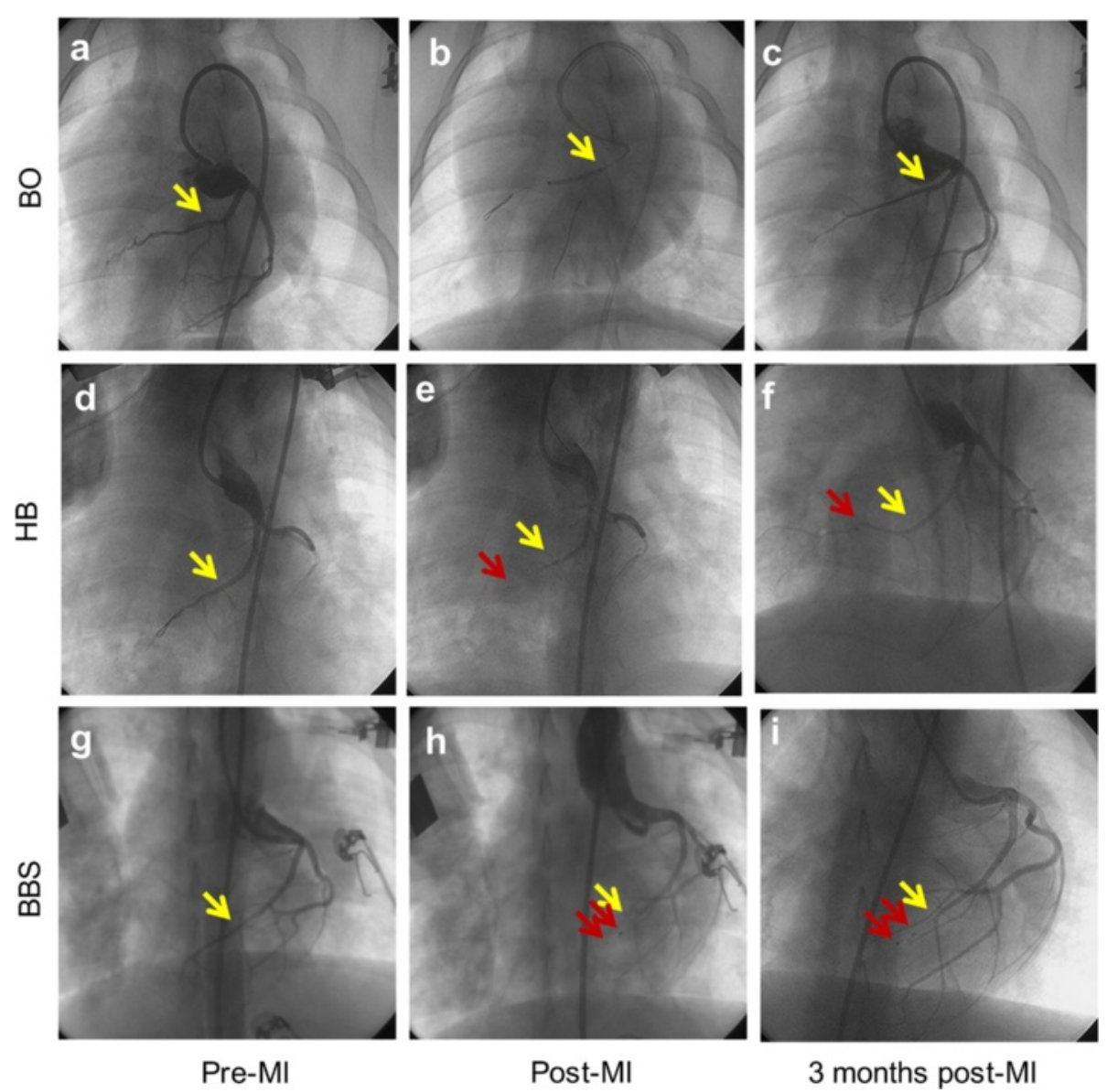

Fig. 3 Presentation and comparison of key operation procedures in three groups. a, d, $\mathbf{g}$ Selective coronary angiography determined the location of balloon occlusion or embolism in three groups. $\mathbf{b}, \mathbf{e}, \mathbf{h}$ Blood flow was completely blocked after balloon occlusion or embolism of the distal LAD coronary artery in three groups, and the blood flow of the second diagonal branch was still normal and clear. $\mathbf{c}, \mathbf{f}, \mathbf{i}$ Three months after operation, coronary angiography confirmed that three minipigs have vascular recanalization in the HB group; meanwhile, no vascular recanalization occurred in the BBS group. The yellow arrow indicates the location of the second diagonal branch, and the red arrows indicate the location of the metal marker of balloon
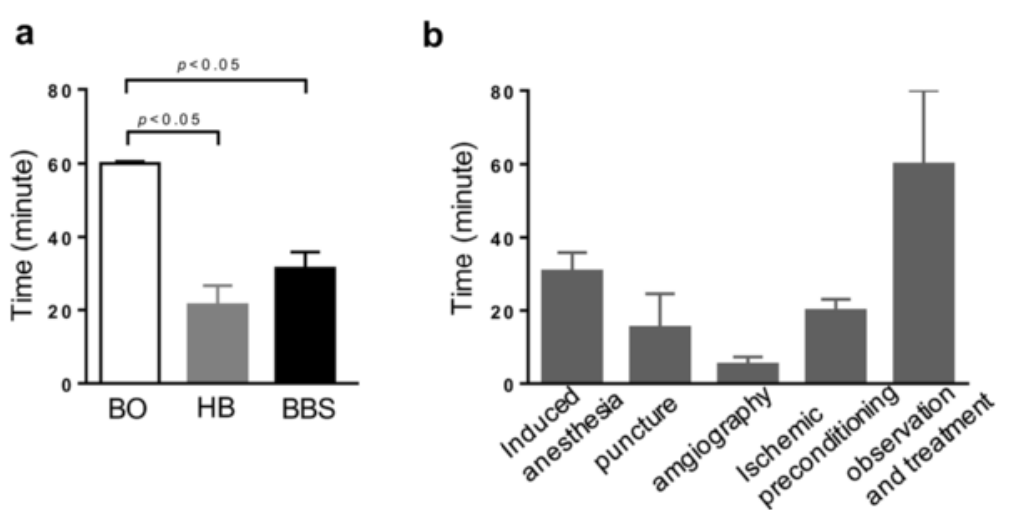

Fig. 4 Comparison of operation time. a Balloon blocking or embolism time in three groups. $\mathbf{b}$ The mean time of each operation procedure in three groups 


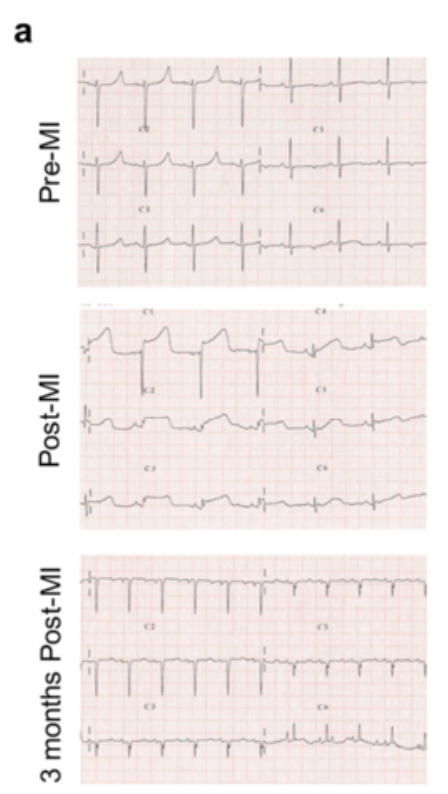

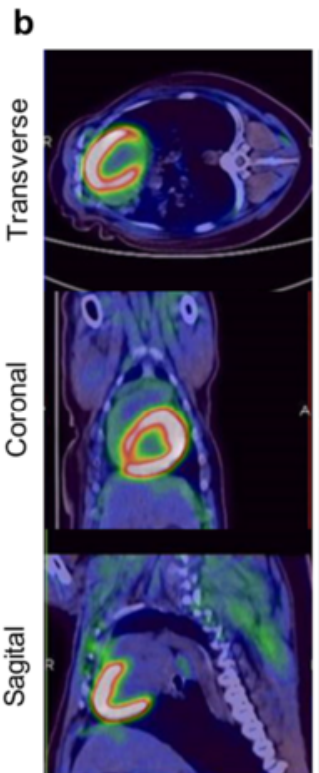

Pre-MI

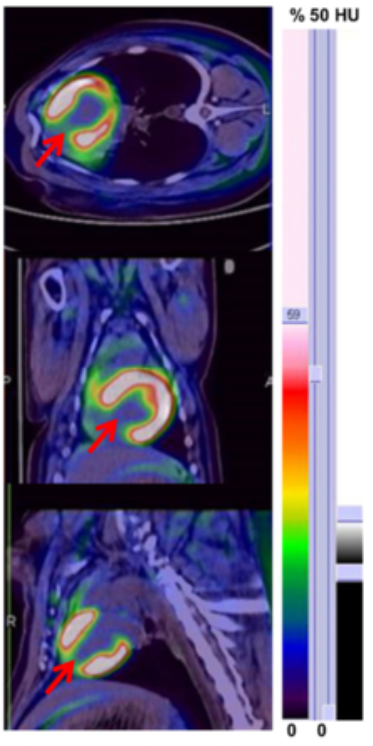

3 months Post $-\mathrm{Ml}$

Fig. 5 Confirmation of myocardial infarction by ECG and ${ }^{18}$ F-FDG PET/CT imaging. a ECG confirmed ST segment of the anterior wall leads dynamic changes in different time points. $\mathbf{b}$ Hybrid imaging of myocardial ${ }^{18} \mathrm{~F}-\mathrm{FDG}$ PET/CT showed that the apical level and anterior wall and anterior septal of mid-level in the BBS group presented myocardial metabolic defect 3 months post-MI. The average standard uptake value (SUV) of region of interest $(\mathrm{ROI})$ indicated by red arrows was $1.50 \pm 0.52$, which was lower than $8.24 \pm 1.29$ with SUVavg of non-ROI $(P<0.05, n=5)$

marker that can be seen in fluoroscopy). After that, the guidewire and the second balloon catheter were withdrawn from the coronary artery. Intermittent coronary angiography was performed, and the time of the complete interruption of blood flow was recorded (Figs. 2e-h and 3d-f).

\section{Sequential balloons-sponge embolism group}

Apart from two balloon catheters, a sponge block (compressible, nonbiodegradable, sterilized, with good biocompatibility) was prepared. The first half-balloon was pushed to $20 \mathrm{~mm}$ distal to the ostium of the second branch of LAD according to the method above. After 10-20 mins, the blood flow of LAD was diminished and completely interrupted. During this time, the second half-balloon was cut and prepared. In turn, the second free half-balloon and 1.5-2.5 mm sponge block and halfballoon with a catheter (similar to a sandwich structure) were set along the guidewire and pushed to $10 \mathrm{~mm}$ distal to the ostium of the second branch of LAD. Following angiography and accurate positioning, the guidewire was withdrawn to the second balloon catheter. During this process, there was no displacement in the two free half-balloon and sponge. After that, the guidewire and the second balloon catheter were withdrawn from the coronary artery. The time of the complete interruption of blood flow was recorded (Figs. 2e-1 and 3g-i).

\section{Coronary angiography and left ventricular angiography} follow-up at 3 months post-MI

Three months after MI, all survived minipigs were subjected to coronary angiography and left ventricular angiography to observe the blood flow condition of LAD and the heart function. Pigtail catheter and high-pressure injector were applied. The procedures were the same as previous procedures.

Evaluation of electrocardiogram, echocardiogram, nuclear medical imaging, and histology staining

Electrocardiogram (ECG) and ultrasonic cardiogram (UCG) were performed prior, immediately, 1 month, and 3 months postoperation. ECG-gated SPECT/CT imaging was performed 1 week before MI and 3 months after MI with a system (SymbiaT2, Siemens, Germany) in three groups. Myocardial perfusion images acquired 1 week before MI was taken as baseline data. Myocardial perfusion images were acquired and semi-quantitative assessment of left ventricular ejection fraction (LVEF) and total perfusion deficit (TPD) were processed as described in our previous study [25]. ${ }^{18}$ F-FDG PET/CT imaging was performed 1 week before $\mathrm{MI}$ and 3 months post-MI in BBS group with an available system (Biograph40, Siemens) to confirm the formation of myocardial infarction. Histological analysis was performed 3 months post-MI. These classic methods have been fully described by previous investigators and introduced in our Additional file 1 [26, 27]. 

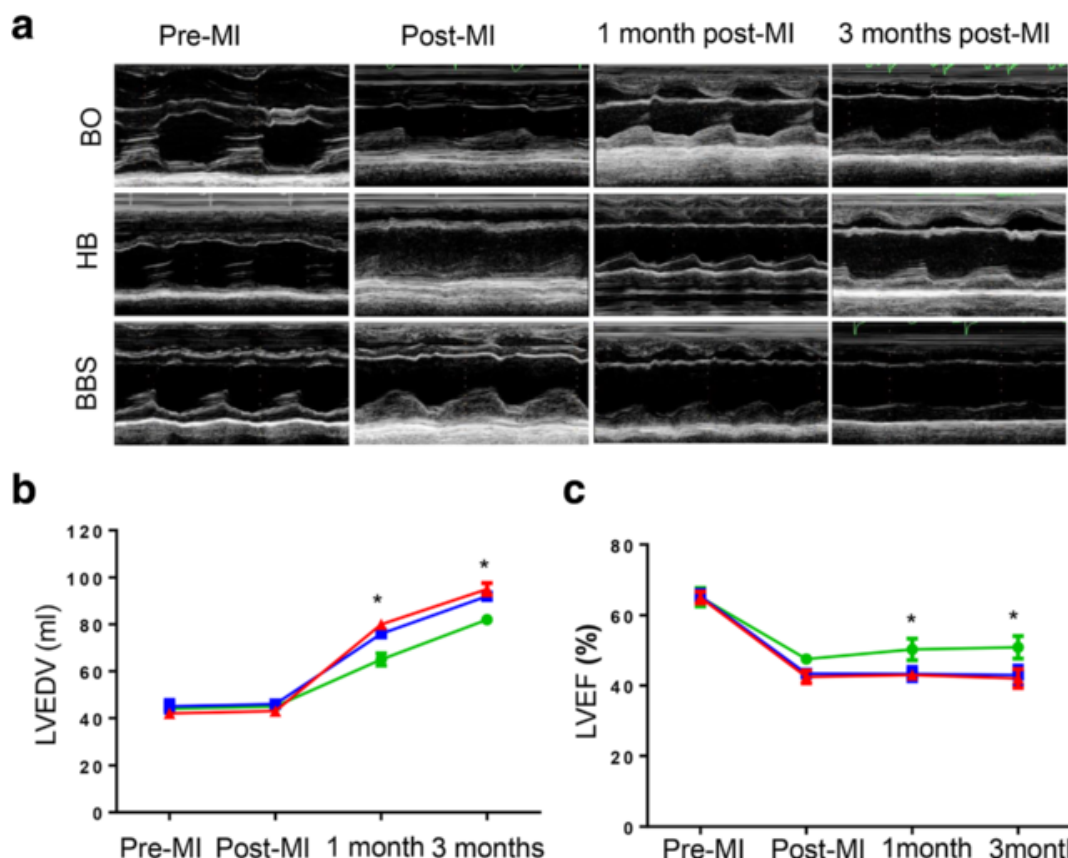

C

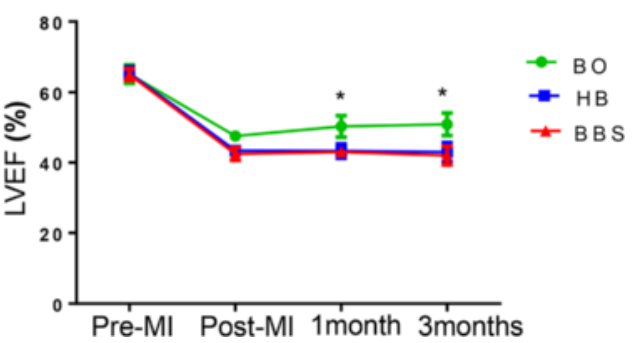

Fig. 6 Echocardiographic evaluation of cardiac function. a Representative M-mode echocardiographic data of hearts at different time points in three groups. The dynamic changes of myocardial infarction in the BO group were only observed in the apical level, and MI of the HB group and BBS group could be observed in the mid-level of the left ventricle. b, c Echocardiographic evaluation of cardiac LVEF and LVEDV (*P $<0.05)$

\section{Statistical analysis}

SPSS14.0 (Chicago, USA) was used for data analysis. To identify significant differences in mortality, incidence of VF, and vascular recanalization among the three groups, Fisher exact test was applied. Continuous variables that approximated the normal distribution were expressed as mean \pm standard error (SEM). Multiple group comparisons were performed by one-way analysis of variance (ANOVA) followed by the least significant difference (LSD) $t$ test for post hoc analysis. Comparisons between the two independent groups were analyzed using the Student's $t$ test. $P<0.05$ was considered statistically significant.

\section{Results}

Viability and myocardial infarction reproducibility

Eighteen minipigs all underwent the procedures, while 5 animals died as a result of intractable VF (3 animals of the $\mathrm{BO}$ group, 1 in the $\mathrm{HB}$ group, and 1 in the BBS group) with an $83.3 \%$ (5/6 pigs) acute procedural survival rate in the $\mathrm{HB}$ group and the BBS group. Incidence of VF and total mortality in the $\mathrm{HB}$ group and BBS group was markedly reduced as compared with the BO group $(P<0.05, n=18)$. Balloon blocking or embolism time was $60.0 \pm 0.5,21.4 \pm 5.2$, and $31.2 \pm 4.7$ mins in the $\mathrm{BO}$ group, $\mathrm{HB}$ group, and BBS group, respectively $(P<0.05)$ (Fig. 4a). In addition, percutaneous femoral artery puncture was performed for a total of 31 times in this study with the operation time around $15.4 \pm$ 6.2 mins. Ultrasound assistance was used once only. The mean time of each procedure in the operation of three groups was presented in Fig. 4b. In general, the entire procedure of each minipig can be achieved within $2-3 \mathrm{~h}$. The follow-up of coronary angiography 3 months after operation revealed that three minipigs in the $\mathrm{HB}$ group appeared to have vascular recanalization, meanwhile none happened in the BBS group $(P<0.05, n=10)$.

\section{Confirmation of myocardial infarction by ECG, UCG, and ${ }^{18}$ F-FDG PET/CT imaging}

The dynamic ST-segment elevation of the anterior wall leads was observed during the procedure (Fig. 5a). ${ }^{18} \mathrm{~F}$ FDG PET/CT imaging showed that myocardial metabolic defect was presented in the myocardium of apical level, anterior wall, and anterior septal in the BBS group (Fig. 5b). Baseline LVEF and the left ventricular enddiastolic volumes (LVEDV) values in UCG were equivalent in the three groups. After MI, there was a significant reduction in LVEF, which is different among the three groups. The LVEF of the BO group at pre-MI, immediately post-MI, 1 month, and 3 months post-MI were $65.2 \pm 2.6,47.6 \pm 1.4,52.3 \pm 3.1$, and $52.9 \pm 3.2 \%$. The values of LVEF in the HB group at the same time points were $65.4 \pm 1.8,43.4 \pm 1.2,43.3 \pm 2.0$, and $43.0 \pm 2.7 \%$, respectively. And the LVEF in the BBS group were 65.0 $\pm 1.7,42.5 \pm 1.8,43.0 \pm 1.2$, and $42.0 \pm 2.7 \%$ at pre-MI, immediately post-MI, 1 month, and 3 months post-MI. The LVEF in the HB group and BBS group was significantly decreased at 1 month, and 3 months post-MI as 


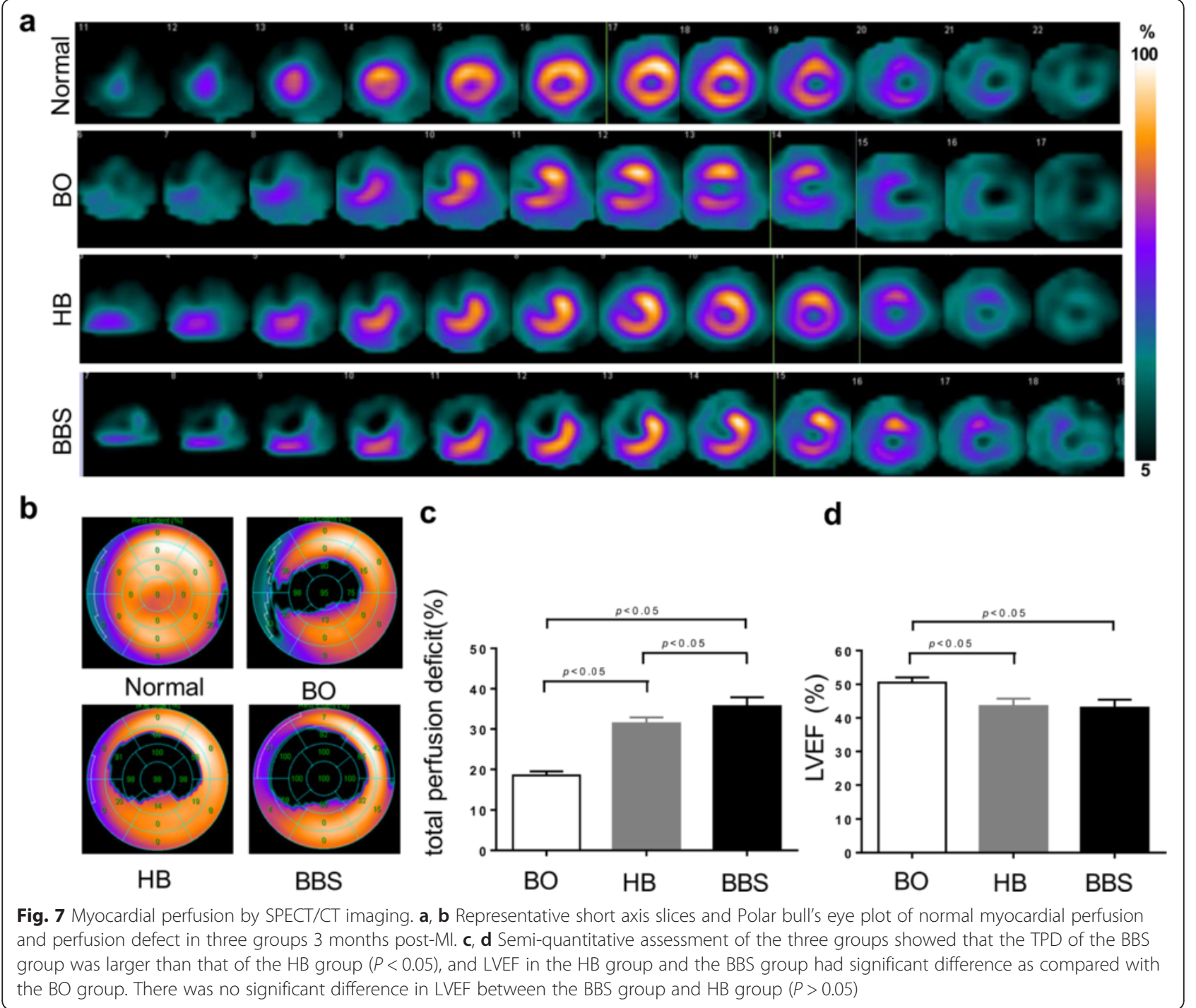

compared with the $\mathrm{BO}$ group $(P<0.05)$. Meanwhile, the LVEDV in the $\mathrm{HB}$ group and BBS group increased higher than that in the $\mathrm{BO}$ group $(76.0 \pm 2.0$ and $80.0 \pm$ 2.2 vs $65.0 \pm 2.7$ at 1 month post-MI, $P<0.05 ; 92.0 \pm 1.7$ and $95.0 \pm 2.7$ vs $82.0 \pm 2.2$ at 3 months post-MI, $P<$ $0.05)$. There was no significant difference in LVEF or LVEDV between the HB group and BBS group $(P>0.05)$ (Fig. 6a-c). Two movies of the left ventricular angiography in the BBS group at pre-MI and 3 months postMI were provided in Additional files 2 and 3, respectively.

\section{Lowest myocardial perfusion in BBS group}

After 3 months post-MI, all the survival animals showed a significant perfusion defect in the apical level and anterior and anterior septal of mid-level in the left ventricle (Fig. 7a). The TPD in the BO group, HB embolism group, and BBS group were $18.5 \pm 1.0,35.4 \pm 1.5$, and
$39.7 \pm 1.2 \%$, respectively. There were significant differences in all the three groups. Especially, the TPD of the BBS group was larger than the $\mathrm{HB}$ group $(P<0.05, n=10)$ (Fig. 7b, c). In addition, the LVEF in the three groups were $50.5 \pm 1.6,43.5 \pm 2.2$, and $43.0 \pm 2.4 \%$, respectively. LVEF in the HB group and BBS group calculated from SPECT data had significant differences as compared with the $\mathrm{BO}$ group, which is similar to the results from echocardiogram. There appeared no significant difference in ejection fraction between the BBS group and HB group $(P>0.05)$ (Fig. $7 \mathrm{~d})$.

Larger infarct size in embolism groups than the BO group Infarct patterns of these three groups were shown by representative triphenyl tetrazolium chloride (TTC) staining (Fig. 8a). The proliferation of fibrous tissue in the infarct zone was further confirmed by Picro Sirius red staining and polarized light observation (Fig. 8b). The infarct size (expressed as LV infarction, \%) in the 


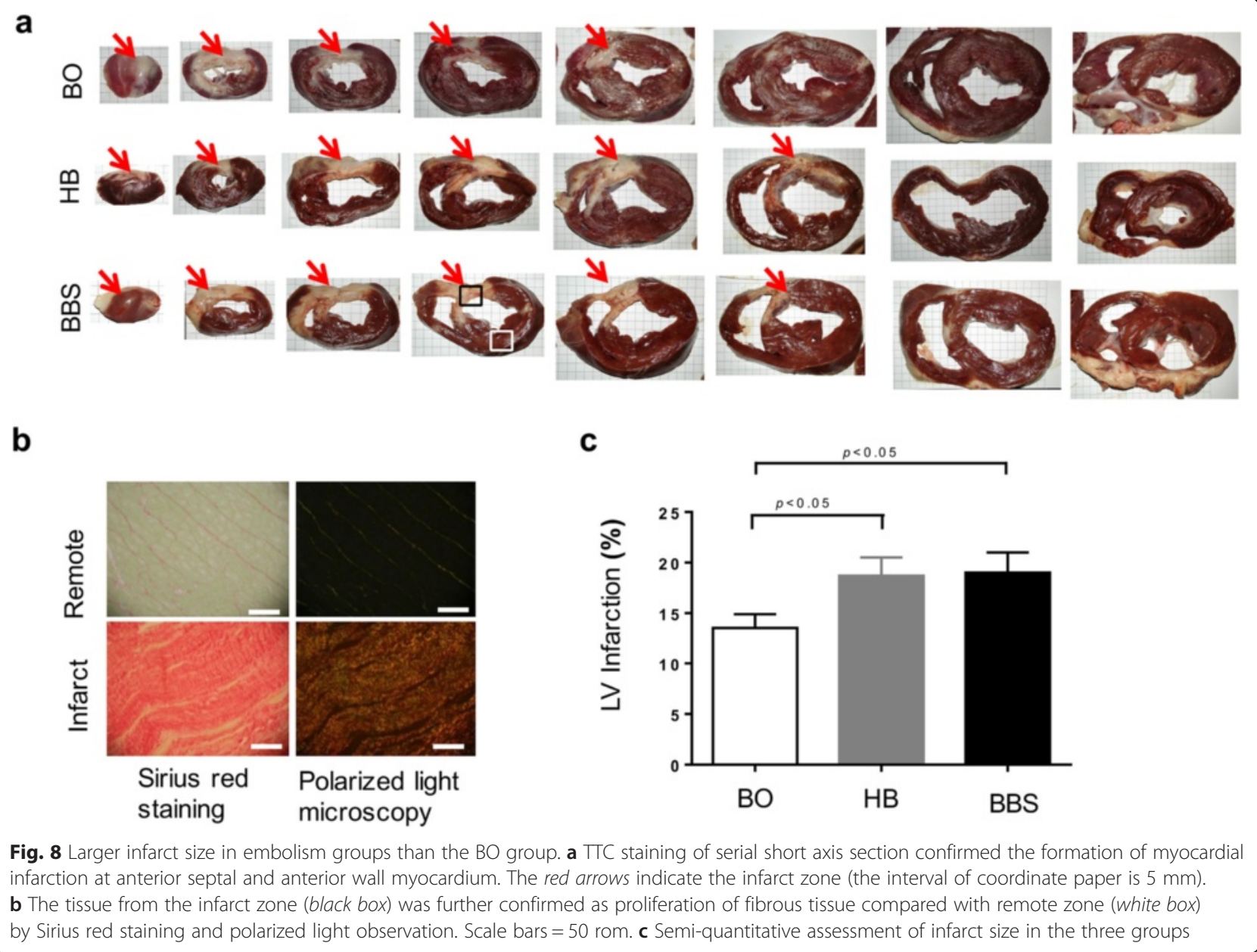

BO group, HB group, and BBS group were $13.5 \pm 1.4$, $18.7 \pm 1.8$, and $19.0 \pm 2.0 \%$, respectively. The HB group and BBS group witnessed significant difference compared with the BO group $(P<0.05)$. There was no significant difference between the two embolism groups $(P>0.05)$ (Fig. 8c).

\section{Discussion}

Porcine MI model has gained popularity due to greater similarity in coronary anatomy and cardiac physiology with humans [28]. Especially, minipig is an ideal experimental animal with smaller body size and genetic stability. In the present study, we set up a new convenient and effective method for chronic LAD embolization by BBS in minipigs. In a previous study, Reffelmann et al. and Dariolli et al. $[14,17]$ had proposed that the sterile sponge can be advanced directly into coronary artery by a balloon catheter to induce MI. However, the sponge is easy to shift and also be off load from the guidewire because of the fusiform structure of the balloon tip. In our study, immediate satisfactory results can be achieved when target vessel was embolized by half-balloon and subsequent thrombosis. However, we found that even though immediate results were satisfactory, LAD recanalization was high during the follow-up. This recovered forward blood flow will lead to misevaluation for the effectiveness of subsequent interventions in the animal model. According to our results, the myocardial perfusion in the BBS group is poorer than that in the $\mathrm{HB}$ group at 3 months post-MI, which may be related to the recanalization in the $\mathrm{HB}$ group. Meanwhile, other methods did not detect the difference between the groups.

In the BBS group with sequential embolism, the metal marker of the balloon can be used to precisely locate the embolism position to keep the consistent infarct size. Subsequent thrombus gradually formed, and the blood flow occluded totally within about 10-20 mins, which can be considered as ischemic preconditioning to protect from acute myocardial injury. This might be the reason for the significant reduction of VF caused by sudden and complete blockage of blood flow. In addition, the second free half-balloon, sponge, and half-balloon with catheter formed a sandwich-like structure, which can be pushed easily to the target position. This sequential embolization 
method maximized the effectiveness and achieved the purpose of complete and permanent occlusion. Meanwhile, the BBS group did not significantly increase the operation time and incidence of VF, which made the chronic MI model more conducive to establish.

As for the evaluation for the chronic MI, heart function, and left ventricular remodeling, several imaging modalities including echocardiography, SPECT/CT, and PET/CT were applied in preclinical study [26, 27]. However, each imaging technique holds its strength and weakness. In order to fully evaluate the whole aspect of the infarcted heart, we applied cardioangiography, echocardiography, SPECT/CT, and ${ }^{18}$ F-FDG PET/CT. Data showed that the infarct size and embolized artery were more stable and repeatable in the BBS group than that in other groups. As a result, the chronic MI model is more consistent and conducive as compared with others. Furthermore, due to the shorter operation time than conventional balloon blocking, the minipig could better tolerate operation. Subsequently, animals were given oxygen inhalation with the nasal catheter and without the tracheal intubation during the operation, which made the experiment simpler and more convenient.

Although this methodology appears to have significant utility for animal research in chronic myocardial infarctions, it is recognized that several study limitations exist. Due to the consideration of experimental cost, the group of 60-min balloon occlusion was established as the only conventional control group, and ${ }^{18} \mathrm{~F}$-FDG PET/CT myocardial metabolism imaging was only used in the BBS group to verify the formation of myocardial infarction.

\section{Conclusions}

Compared to the conventional modeling induced by balloon blocking, the method of sequential balloonssponge embolization could induce myocardial infarction with consistent and sustained embolization and gain higher operation success rate and better repeatability in minipigs, which holds a promising method for preclinical myocardial infarction study.

\section{Additional files}

Additional file 1: Classic methods have been fully described by previous investigators and introduced. (DOCX $29 \mathrm{~kb}$ )

Additional file 2: Left ventricular angiography of pre-MI in BBS group. (AVI $2406 \mathrm{~kb}$ )

Additional file 3: Left ventricular angiography of 3 months post-MI in BBS group. (AVI $2427 \mathrm{~kb}$ )

\section{Abbreviations}

ECG, electrocardiogram; EDV, end-diastolic volumes; LAD, left anterior descending; LVEF, left ventricular ejection fraction; MI, myocardial infarction; $\mathrm{PET} / \mathrm{CT}$, positron emission tomography/computed tomography; PTCA, percutaneous transluminal coronary angioplasty; SPECT, single-photon emission computed tomography; TTC, triphenyl tetrazolium chloride; UCG, ultrasonic cardiogram; VF, ventricular fibrillation

\section{Funding}

This study was funded by grants from the National Distinguished Young Scientists Grant of China (81325009) and National Nature Science Foundation of China (No. 81270168, 81530058), (FCao BWS12J037), Innovation Team Development Grant by China Department of Education (IRT1053), and National Basic Research Program of China (2012CB518101).

\section{Authors' contributions}

The study of large animal need multi-disciplinary cooperation. In our study, BT and HKG designed the experiment, conducted the operation personally and drafted the manuscript. ZHL, SM and YBW did assisted work in these process. Professor MWZ, LWL, JW, LG, and FC proposed valuable comments on the improvements of $\mathrm{MI}$ model and revising the manuscript critically for important intellectual content. In addition, WB and DLL did a lot of work in the imaging examination and data analysis. All authors read and approved the final manuscript.

\section{Competing interests}

The authors declare that they have no competing interests.

\section{Consent for publication}

Not applicable.

\section{Ethical approval}

All applicable international, national, and/or institutional guidelines for the care and use of animals were followed. This article does not describe any studies with human participants performed by any of the authors.

\section{Author details}

'Department of Cardiology, Chinese PLA General Hospital, Fuxing Street 28\#, Haidian District, Beijing 100853, China. ${ }^{2}$ Department of Cardiology, Xijing Hospital, Fourth Military Medical University, Xi'an 710032, China. ${ }^{3}$ Department of Radiology, Xijing Hospital, Fourth Military Medical University, Xi'an 710032, China. ${ }^{4}$ Department of Ultrasonography, Xijing Hospital, Fourth Military Medical University, Xi'an 710032, China. ${ }^{5}$ Department of Nuclear Medicine, Xijing Hospital, Fourth Military Medical University, Xi'an 710032, China.

Received: 23 April 2016 Accepted: 29 June 2016

Published online: 08 July 2016

\section{References}

1. GBD 2013 Mortality and Causes of Death Collaborators. Global, regional, and national age-sex specific all-cause and cause-specific mortality for 240 causes of death, 1990-2013: a systematic analysis for the Global Burden of Disease Study 2013. Lancet. 2015;385(9963):117-71.

2. Mozaffarian D, Benjamin EJ, Go AS, Arnett DK, Blaha MJ, Cushman M, et al. Heart disease and stroke statistics - 2015 update: a report from the American Heart Association. Circulation. 2015;131(4):e29-322.

3. Gavira JJ, Nasarre E, Abizanda G, Perez-Ilzarbe M, de Martino-Rodriguez A, Garcia de Jalon JA, et al. Repeated implantation of skeletal myoblast in a swine model of chronic myocardial infarction. Eur Heart J. 2010;31(8):1013-21.

4. Davies M, Hobbs F, Davis R, Kenkre J, Roalfe AK, Hare R, et al. Prevalence of left-ventricular systolic dysfunction and heart failure in the

Echocardiographic Heart of England Screening study: a population based study. Lancet. 2001;358(9280):439-44.

5. McCall FC, Telukuntla KS, Karantalis V, Suncion VY, Heldman AW, Mushtaq $M$, et al. Myocardial infarction and intramyocardial injection models in swine. Nat Protoc. 2012;7(8):1479-96.

6. Malliaras K, Smith RR, Kanazawa H, Yee K, Seinfeld J, Tseliou E, et al. Validation of contrast-enhanced magnetic resonance imaging to monitor regenerative efficacy after cell therapy in a porcine model of convalescent myocardial infarction. Circulation. 2013;128(25):2764-75.

7. Quevedo HC, Hatzistergos KE, Oskouei BN, Feigenbaum GS, Rodriguez JE, Valdes $D$, et al. Allogeneic mesenchymal stem cells restore cardiac function in chronic ischemic cardiomyopathy via trilineage differentiating capacity. Proc Natl Acad Sci U S A. 2009:106(33):14022-7.

8. de Silva R, Raval AN, Hadi M, Gildea KM, Bonifacino AC, Yu ZX, et al. Intracoronary infusion of autologous mononuclear cells from bone marrow or granulocyte colony-stimulating factor-mobilized apheresis product may not 
improve remodelling, contractile function, perfusion, or infarct size in a swine model of large myocardial infarction. Eur Heart J. 2008;29(14):1772-82.

9. Krombach GA, Kinzel S, Mahnken AH, Gunther RW, Buecker A. Minimally invasive close-chest method for creating reperfused or occlusive myocardial infarction in swine. Invest Radiol. 2005;40(1):14-8.

10. Li X, Shao D, Wang G, Jiang T, Wu H, Gu B, et al. Effects of different LAD-blocked sites on the development of acute myocardial infarction and malignant arrhythmia in a swine model. J Thorac Dis. 2014;6(9):1271-7.

11. de Jong R, van Hout GP, Houtgraaf JH, Takashima S, Pasterkamp G, Hoefer I, et al. Cardiac function in a long-term follow-up study of moderate and severe porcine model of chronic myocardial infarction. BioMed Res Int. 2015;2015:209315.

12. Dib N, Diethrich EB, Campbell A, Gahremanpour A, McGarry M, Opie SR. A percutaneous swine model of myocardial infarction. J Pharmacol Toxicol Methods. 2006;53(3):256-63.

13. Kim W, Jeong MH, Sim DS, Hong YJ, Song HC, Park JT, et al. A porcine model of ischemic heart failure produced by intracoronary injection of ethyl alcohol. Heart Vessels. 2011;26(3):342-8.

14. Reffelmann T, Sensebat O, Birnbaum Y, Stroemer E, Hanrath P, Uretsky BF, et al. A novel minimal-invasive model of chronic myocardial infarction in swine. Coron Artery Dis. 2004;15(1):7-12.

15. Crisostomo V, Maestre J, Maynar M, Sun F, Baez-Diaz C, Uson J, et al. Development of a closed chest model of chronic myocardial infarction in Swine: magnetic resonance imaging and pathological evaluation. ISRN Cardiol. 2013;2013:781762.

16. Hanes DW, Wong ML, Jenny Chang CW, Humphrey S, Grayson JK, Boyd WD, et al. Embolization of the first diagonal branch of the left anterior descending coronary artery as a porcine model of chronic trans-mural myocardial infarction. J Transl Med. 2015;13:187.

17. Dariolli R, Takimura CK, Campos CA, Lemos PA, Krieger JE. Development of a closed-artery catheter-based myocardial infarction in pigs using sponge and lidocaine hydrochloride infusion to prevent irreversible ventricular fibrillation. Physiol Rep. 2014;2(8):e12121.

18. Spata T, Bobek D, Whitson BA, Parthasarathy S, Mohler PJ, Higgins RS, et al. A nonthoracotomy myocardial infarction model in an ovine using autologous platelets. BioMed Res Int. 2013;2013:938047.

19. Biondi-Zoccai G, De Falco E, Peruzzi M, Cavarretta E, Mancone M, Leoni O, et al. A novel closed-chest porcine model of chronic ischemic heart failure suitable for experimental research in cardiovascular disease. BioMed Res Int. 2013;2013:410631.

20. Peukert D, Laule M, Kaufels N, Schnorr J, Taupitz M, Hamm B, et al. A minimally invasive method for induction of myocardial infarction in an animal model using tungsten spirals. Int J Cardiovasc Imaging. 2009;25(5):529-35.

21. Munz MR, Faria MA, Monteiro JR, Aguas AP, Amorim MJ. Surgical porcine myocardial infarction model through permanent coronary occlusion. Comp Med. 2011;61(5):445-52.

22. Suzuki Y, Lyons JK, Yeung AC, Ikeno F. In vivo porcine model of reperfused myocardial infarction: in situ double staining to measure precise infarct area/area at risk. Catheter Cardiovasc Interv. 2008:71(1):100-7.

23. Contamin H, Rioufol G, Bettinger T, Helbert A, Portier KG, Lepage OM, et al. A minimally-invasive closed chest myocardial occlusion-reperfusion model in rhesus monkeys (Macaca mulatta): monitoring by contrast-enhanced ultrasound imaging. Int J Cardiovasc Imaging. 2012;28(3):531-42.

24. Lukacs E, Magyari B, Toth L, Petrasi Z, Repa I, Koller A, et al. Overview of large animal myocardial infarction models (review). Acta Physiol Hung. 2012;99(4):365-81.

25. Tao B, Cui M, Wang C, Ma S, Wu F, Yi F, et al. Percutaneous intramyocardial delivery of mesenchymal stem cells induces superior improvement in regional left ventricular function compared with bone marrow mononuclear cells in porcine myocardial infarcted heart. Theranostics. 2015;5(2):196-205.

26. Weidemann F, Dommke C, Bijnens B, Claus P, D'Hooge J, Mertens P, et al. Defining the transmurality of a chronic myocardial infarction by ultrasonic strain-rate imaging: implications for identifying intramural viability: an experimental study. Circulation. 2003;107(6):883-8.

27. Lautamaki R, Schuleri KH, Sasano T, Javadi MS, Youssef A, Merrill J, et al. Integration of infarct size, tissue perfusion, and metabolism by hybrid cardiac positron emission tomography/computed tomography: evaluation in a porcine model of myocardial infarction. Circ Cardiovasc Imaging. 2009;2(4):299-305.

28. Suzuki Y, Yeung AC, Ikeno F. The representative porcine model for human cardiovascular disease. J Biomed Biotechnol. 2011;2011:195483.

\section{Submit your manuscript to a SpringerOpen ${ }^{\circ}$ journal and benefit from:}

- Convenient online submission

- Rigorous peer review

- Immediate publication on acceptance

- Open access: articles freely available online

High visibility within the field

- Retaining the copyright to your article

Submit your next manuscript at $\gg$ springeropen.com 